\title{
Vascular atrophy and VEGFR-2 signaling: old theories of pulmonary emphysema meet new data
}

\author{
Steven D. Shapiro
}

Division of Pediatric Allergy and Pulmonary Medicine, Departments of Pediatrics, Medicine, and Cell Biology,

Washington University School of Medicine at St. Louis Children's and Barnes-Jewish Hospital, 660 S. Euclid Avenue,

St. Louis, Missouri 63110, USA. Phone: (314) 286-2778; Fax: (314) 286-2895; E-mail: shapiro_s@kids.wustl.edu.

Pulmonary emphysema, defined as airspace enlargement distal to terminal bronchioles, is a major component of chronic obstructive pulmonary disease (COPD). COPD is currently the fourth leading cause of death in the US, with a worldwide epidemic looming as an addicted humankind enthusiastically lights up its cigarettes. To make matters worse, short of lung transplantation and volume reduction surgery, we have little to offer these patients, other than oxygen, as they grasp for each breath. Improved understanding of the biological basis of this disease is required if we hope to intervene.

Two observations made in the early 1960s, one clinical and one experimental, form the cornerstone of our current understanding of emphysema. First, Laurell and Eriksson reported an association of chronic airflow obstruction and emphysema with deficiency of serum $\alpha_{1}$-antitrypsin $\left(\alpha_{1}\right.$-AT), the inhibitor of the serine proteinase neutrophil elastase (NE) (1). Second, Gross et al. developed the first reproducible model of emphysema, in which they injected the plant protease papain into the lungs of experimental animals (2). Together these two observations indicated that emphysema could be induced by proteolytic injury to the lung ECM, and they led to the elastase/antielastase hypothesis for the pathogenesis of emphysema. Prior to this time, mechanical explanations for emphysema dominated, although, as early as 1905, Opie had suggested that enzymes and antienzymes determined the risk of emphysema (3). Another largely forgotten hypothesis was Liebow's vascular atrophy model for emphysema, proposed in the 1950s (4). In this issue of the JCI, Tuder and colleagues (5) revive this hypothesis armed with modern concepts of endothelial cell biology and cell death.
In order to appreciate the novelty of the findings presented by Tuder and colleagues. (5), one must understand how the elastase/antielastase hypothesis has evolved over the last 35 years. It is generally accepted that chronic exposure to cigarette smoke leads to recruitment of inflammatory cells into the terminal airspaces of the lung, where they release elastolytic proteinases in excess of inhibitors in local microenvironments, damaging the ECM of the lung. Ineffective repair of alveoli and elastic fibers and perhaps other ECM components results in airspace enlargement that defines pulmonary emphysema. We continue to struggle to determine the contribution of small airway changes and airspace enlargement to loss of lung function in COPD.

Within this general framework, research has concentrated on which inflammatory cells and proteinases are most relevant. Macrophages predominate in the lungs of smokers, but neutrophils and $T$ cells also accumulate in COPD. The importance of NE in the pathogenesis of emphysema associated with $\alpha_{1}$-AT deficiency has led the field to assume that this proteinase plays a similar role in more common forms of emphysema. However, work in transgenic and gene-targeted mouse models of the disease suggests that macrophage MMPs may also be important. Thus, mice deficient in macrophage elastase (MMP-12) fail to develop emphysema when exposed to long-term cigarette smoke (6), and overexpression of the interstitial collagenase MMP-1 in the lungs of transgenic mice also leads to airspace enlargement (7). Whether the latter phenotype reflects an event during the pathogenesis of emphysema or merely represents a developmental abnormality, this finding highlights the potential importance of matrix components other than elastin. Clearly, when an alveolar space is destroyed, all ECM components are lost, including collagen, which diffusely surrounds the airspace, elastin, which is concentrated on the openings to the alveolus, and type IV collagen, entactin, laminin, and proteoglycans, components of the basement membrane that separates endothelial and epithelial cells. However, in emphysematous lungs, total collagen content is increased, and collagen deposits in small airways predispose to airflow obstruction (8). Thus, the role of collagen turnover is complex and compartment-specific.

The fact that only $15-20 \%$ of cigarette smokers develop emphysema suggests that other genetic and environmental factors interact with cigarette smoke to cause emphysema. One might speculate that abnormalities affecting inflammation, the balance of proteinases and antiproteinases, or the lung's ability to repair tissue injury could all predispose to emphysema. Hogg and colleagues suggest that latent adenoviral infection augments the inflammatory response in susceptible individuals (9). In addition to $\alpha_{1}$-AT deficiency, perhaps imbalance of other proteinases and inhibitors leads to emphysema. Alternatively, smoking might lead to predictable inflammation and proteolytic damage, and inability to adequately repair the alveolus might determine those susceptible to emphysema. Use of inbred strains of mice, and identification of non- $\alpha_{1}$-AT families with strong predisposition to emphysema, will help us sort out genetic factors and hence pathogenetic mechanisms.

Ultimately, if we hope to cure the disease we need to repair damaged lung tissue. The finding by the Massaro and Massaro (10) that retinoic acid reverses pancreatic elastase-induced emphysema in rats provides the first evidence that new alveolar formation is possible 
upon an emphysematous substrate. This therapy is currently undergoing human trials. However, even if this strategy is unsuccessful, we must build upon the clues provided by this phenomenon. Intense investigation of the processes involved in normal lung development should lead to strategies for repair in disease.

In the current study by Tuder and colleagues in this issue of the JCI (5), the authors applied a VEGFR-2 inhibitor to rat lungs, causing endothelial and epithelial apoptosis followed by airspace enlargement. This simple but elegant finding operates entirely outside the concept of inflammatory destruction causing emphysema, forcing us to rethink these fundamental concepts (Figure 1). Traditionally, we think of airspace enlargement occurring through destruction of the basement membrane and supporting structures by proteinases. Loss of cell attachment to the basement membrane is then presumed to cause the death of surrounding epithelial and endothelial cells. In the model proposed here, cell death initiates the process. The article does not address how this cell death leads to loss of the ECM (5), but Tuder and colleagues state that this process is noninflammatory, implying that proteinases released by endothelial and/or epithelial cells are sufficient to cause ECM destruction. MMPs and cathepsins expressed in these cell types are the most logical candidates, although

\section{Figure 1}

Mechanisms of airspace enlargement in pulmonary emphysema. The traditional inflammatory cell hypothesis stipulates that cigarette smoke results in accumulation of inflammatory cells that release proteinases (neutrophil elastase [NE], macrophage elastase [MMP-12], and cysteine proteinases) disrupting extracellular matrix and basement membrane. Loss of cell-matrix attachment leads to apoptosis with loss of the entire alveolar unit. Proposed here is an alternative hypothesis whereby cell death is the primary stimulus with subsequent loss of matrix components resulting in loss of the alveolar unit. one cannot exclude other intracellular proteinases such as caspases, which have never been tested for ECM-degrading capacity.

Like most paradigm shifts, this study generates more questions than it answers. What is the specificity of VEGFR-2 inhibition? The authors believe that endothelial cells are specifically targeted. However, in a recent issue of the JCI, Dias et al. reported finding VEGFR2 expressed in hematopoietic cells (11). Perhaps inflammatory cell death with proteinase release plays a greater role here than anticipated. Alternatively, epithelial cells may be directly influenced by this inhibitor. How will these findings influence VEGFR-2 treatment for cancer? These data suggest that normal vessels require VEGF signaling to maintain their integrity. Will VEGFR-2 inhibitors cure cancer but cause emphysema? Finally, what is the relevance of this model to human COPD caused by cigarette smoking? Tuder and colleagues (5) have presented preliminary data associating loss of VEGFR-2 expression with COPD. Regardless of the actual role of VEGFR-2 in COPD, this article prompts us to think more freely about mechanisms of airspace enlargement. For example, combining these concepts with other interesting and unexplained findings such as the presence of latent viral infections and the presence of $\mathrm{CD}^{+} \mathrm{T}$ cells associated with emphysema (12), one could postulate that epithelial cell cytotoxicity by
$\mathrm{CD}^{+}$cells also causes airspace enlargement. Given our inability to rid the world of cigarettes and our lack of medical therapy for emphysema, new insights that force us to think are refreshing.

1. Laurell, C.B., and Eriksson, S.E. 1963. The electrophoretic alpha-globulin pattern of serum in alpha-antitrypsin deficiency. Scand. J. Clin. Lab. Invest. 15:132-140.

2. Gross, P., Pfitzer, E., Tolker, E., Babyak, M., and Kaschak, M. 1965. Experimental emphysema: its production with papain in normal and silicotic rats. Arch. Environ. Health. 11:50-58.

3. Opie, E. 1905. Enzymes and anti-enzymes of inflammation exudates. J. Exp. Med. 7:316-334.

4. Liebow, A. 1959. Pulmonary emphysema with special emphasis to vascular changes. Am. Rev. Respir. Dis. 80:67-93.

5. Kasahara, Y., et al. Inhibition of VEGF receptors causes lung cell apoptosis and emphysema. 2000. J. Clin. Invest. 106:1311-1319.

6. Hautamaki, R.D., Kobayashi, D.K., Senior, R.M., and Shapiro, S.D. 1997. Macrophage elastase is required for cigarette smoke-induced emphysema in mice. Science. 277:2002-2004.

7. D’Armiento, J., Dalal, S.S., Okada, Y., Berg, R.A., and Chada, K. 1992. Collagenase expression in the lungs of transgenic mice causes pulmonary emphysema. Cell. 71:955-961.

8. Wright, J.L. 1995. Emphysema: concepts under change. A pathologist's perspective. Mod. Pathol. 8:873-880.

9. Keicho, N., Elliot, N., Hogg, J., and Hayshi, S. 1997. Adenovirus E1A upregulates interleukin-8 expression induced by endotoxin in pulmonary epithelial cells. Am. J. Physiol. 272:L1046-L1052.

10. Massaro, G.D., and Massaro, D. 1997. Retinoic acid treatment abrogates elastase-induced pulmonary emphysema in rats. Nat. Med. 6:675-677.

11. Dias, S., et al. 2000. Autocrine stimulation of VEGFR-2 activates human leukemic cell growth and migration. J. Clin. Invest. 106:511-521.

12. Saetta, M., et al. 1998. CD8 T-lymphocytes in peripheral airways of smokers with chronic obstructive pulmonary disease. Am. J. Respir. Crit. Care Med. 157:822-826.

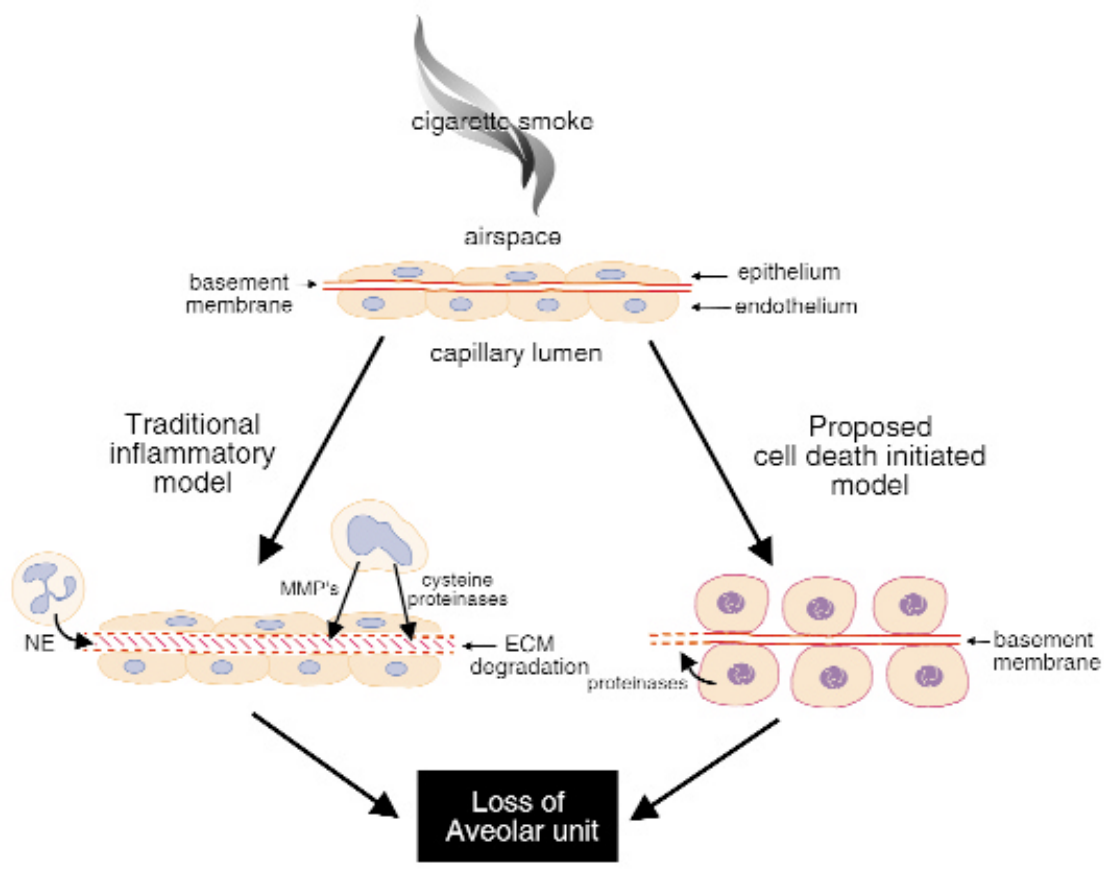

